EPJ Web of Conferences 66, 01014 (2014)

DOI: $10.1051 /$ epjconf/ 20146601014

(C) Owned by the authors, published by EDP Sciences, 2014

\title{
Direct reactions with exotic nuclei
}

\author{
A. Obertelli
}

${ }^{1}$ CEA, Centre de Saclay, IRFU, F-91191 Gif-sur-Yvette, France

\begin{abstract}
Direct reactions have been a unique tool to address the nuclear many-body problem from the experimental side. They are now routinely used in inverse kinematics with radioactive ion beams (RIB). However, weakly bound nuclei have recently raised questions on the applicability of reaction formalisms benchmarked on stable nuclei to the study of single-particle properties and correlations in these unstable systems. The study of the most exotic species produced at low intensity have triggered new technical developments to increase the sensitivity of the setup, with a focused attention to direct reactions such as transfer at low incident energy or knockout at intermediate energies.
\end{abstract}

\section{Nuclear structure from direct reactions}

In nuclear reactions, some processes leave the final nucleus in a state that retains recollections of the initial wave function. These reactions, for which few degrees of freedom were modified, are called direct [1]. This gives to direct reactions, in addition to their selectivity, the strong advantage to allow a quantitative investigation of the ground-state properties of atomic nuclei.

Different direct reaction mechanisms are used depending on the incident energy. Often encountered are nucleon transfer reactions at low incident energy, generally in a regime between 5 and 50 $\mathrm{MeV} /$ nucleon, and knockout reactions at relativistic energies, typically above $150 \mathrm{MeV} /$ nucleon to minimize indirect contributions to the direct cross section. The electron-induced stripping reaction (e,e'p) is considered to be the reference stripping measurement from stable nuclei. As an electromagnetic probe, (e,e'p) is considered to be well understood when restricted to large momentum transfer. In this case, small corrections have to be taken into account for final state interaction with the proton in the exit channel [2]. Charge density and intrinsic momentum distributions of protons in stable nuclei have been well studied this way. The neutron component of nuclei requires a nuclear probe. Transfer and $(p, 2 p)$ reactions have been benchmarked with (e,e'p) and found to be consistent for stable nuclei when treated as direct mechanisms and analyzed within a DWBA framework [3].

Information extracted from the analysis of direct reaction cross sections has driven our understanding of the nuclear shell structure and their usefulness is unanimously recognized. The spectroscopic strength obtained from pickup and stripping reactions are indeed necessary to quantify the amount of correlations in a given nucleus and offer the possibility to address, when extracted from cross sections within a given theoretical framework, the question of uncorrelated single-particle energies $E_{s p}$ [4]

$$
E_{s p}=\sum_{p} S F_{p}^{+} E_{p}+\sum_{h} S F_{h}^{-} E_{h}
$$

This is an Open Access article distributed under the terms of the Creative Commons Attribution License 2.0, which permits unrestricted use, distribution, and reproduction in any medium, provided the original work is properly cited. 
Where $S F^{+(-)}$are the so-called spectroscopic factors extracted from the pickup (stripping) cross sections. The non-observable nature of spectroscopic factors and single-particle energies restricts their use to being model dependent. However they are still necessary and allow for a depiction of nuclear structure [5,6]. The case of neutron stripping and pickup from ${ }^{40,48} \mathrm{Ca}$ can be considered as a typical example from which the nuclear structure community has built part of its representation of nuclear structure in terms of single-particle excitations and correlations [7-9].

From the early 90s, direct reactions have been widely used as a selective probe for the structure of unstable nuclei. The spectroscopy of radioactive ions can only be reached in inverse kinematics and laboratories such as GANIL, REX-ISOLDE at CERN, RIKEN and the NSCL, have successfully devoted a significant part of their research programs to in-beam spectroscopy in inverse kinematics.

\section{Spectroscopic strength from one-nucleon stripping}

The distribution of spectroscopic strength in nuclei can be extracted from direct-reaction cross section measurements, assuming a modeling of the reaction mechanism.

Recently, a compilation of one-nucleon removal at intermediate energies from sd-shell exotic nuclei showed that the measured cross sections for knocking out a valence nucleon in a very asymmetric nucleus (such as a neutron in ${ }^{32} \mathrm{Ar},{ }^{28} \mathrm{Ar}$ and ${ }^{24} \mathrm{Si}$ ) are about four times smaller than predictions from state-of-the-art calculations [10]. On the other hand, at low energy, a study of the (p,d) neutron transfer on the proton-rich ${ }^{34} \mathrm{Ar}$ and on the neutron-rich ${ }^{46} \mathrm{Ar}$ provides experimental spectroscopic factors in agreement with large-basis shell model calculations to within 20\% [11]. These findings which are in agreement with a previous systematic study of transfer reactions [12] are inconsistent with the trend observed in knockout.Very recently, a new systematic study over transfer reactions with stable nuclei did not evidence any dependence of the effect of short range or beyond model space correlations with the transfered angular momentum $\ell$, mass of target nuclei or assymetry $\Delta \mathrm{S}$ [13]. Therefore, it is suggested that these two probes, transfer and knockout, lead systematically to different spectroscopic factors when analysed in the above mentioned frameworks, namely DBWA or CRC for transfer and under the sudden and eikonal approximations for knockout. The origin of this difference has to be understood.

A recent study of the nucleon removal from ${ }^{14} \mathrm{O}$ and ${ }^{16} \mathrm{C}(\Delta \mathrm{S}=|S n-S p|$ close to $20 \mathrm{MeV})$ at intermediate energies lower than $100 \mathrm{MeV}$ nucleon has shown that the applicability of the sudden approximation and the eikonal theory for nucleon removal depends on both the incident beam energy and the binding energy of the removed nucleon [14]. The applicability of the eikonal formalism to the previously deeply-bound nucleon removal was questioned. Indeed, one may question the rôle of dissipation in deeply-bound nucleon removal [15].

The corresponding transfer stripping reactions $(\mathrm{d}, \mathrm{t})$ and $(\mathrm{d}, 3 \mathrm{He})$ from the same ${ }^{14} \mathrm{O}$ nucleus at $18 \mathrm{MeV} /$ nucleon performed at GANIL [16] and analyzed within the framework of coupled reaction channel formalism with a set of optical potentials, matter radii and spectroscopic factors did not show any systematic reduction for deeply-bond nucleon stripping at variance with [10]. This is in agreement with the analysis of [11] andé[13].

A more systematic study of deeply-bound nucleon removal reactions from weakly bound nuclei will definitely help understanding the limits of current direct reaction models.

\section{Long-range correlations via two nucleon stripping}

Two-body correlations are also a key aspect of nuclear structure. It can be probed via direct reactions. Momentum and spatial correlations in the nucleus have been investigated at all incident energies with 
INPC 2013

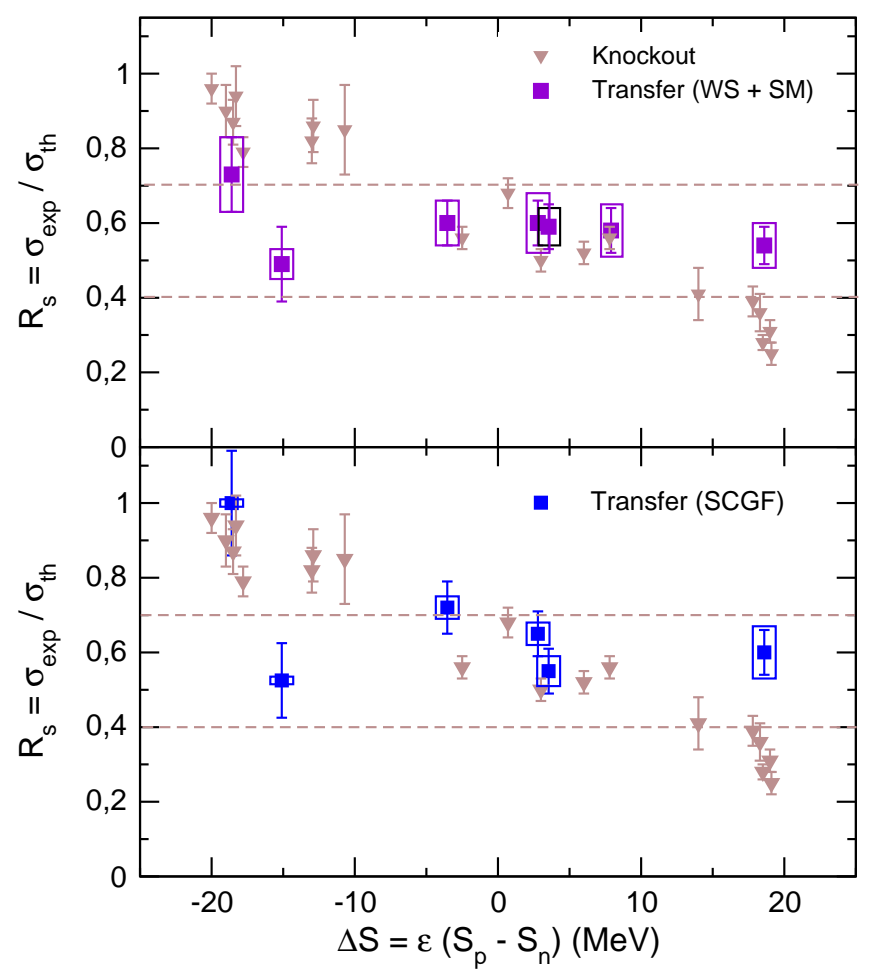

Figure 1. Ratio of experimental to theoretical one-nucleon stripping cross sections. Experimental knockout data from $[10,14]$ are considered and their theoretical predictions are performed within the eikonal formalism under the sudden approximation. Transfer data from oxygen isotopes are taken from [16] and their theoretical preditions are performed within the Coupled Reaction Channel formalism. Analysis with traditional (based on Woods-Saxon single particle wave functions) form factors is are shown in the top panel. The bottom panel shows similar analysis with Self-Consistent Green's Function ab initio form factors.

different reaction mechanisms in the past. The two neutron transfer has been widely used in both direct and indirect kinematics and is known to be sensitive to configuration transitions, such as shape transitions [17] or pairing in light [18, 19] or heavy nuclei [20]. Two-nucleon knockout has been more recently introduced to probe correlations [21, 22] and a dedicated formalism has been developed showing a strong sensitivity to two-body correlations of the intermediate-energy inclusive knockout $[23,24]$. At variance with transfer, the two-nucleon knockout probes only the spatial correlations. The fast two-neutron stripping and two-neutron transfer may then be seen as complementary probes for two-body correlations. Still at intermediate energies, the incomplete transfer or towing mode [25] has been also used to investigate correlations in two neutron halos [26]. At relativistic incident energies, exclusive quasi free scattering with large momentum transfer can be seen as a perfect probe and should be investigated further. A striking example is the case of the ground-state wave-function of ${ }^{11} \mathrm{Li}$ : although this borromean nucleus has been the center of several experimental investigations, there is no consensus on the details of its wave function in terms of the nuetron configurations and the proportion of ${ }^{9} \mathrm{Li}$ in its ground-state and excited state in ${ }^{11} \mathrm{Li}$, assuming the universality of such a shell model representation of the ground state of ${ }^{11} \mathrm{Li}$. In the case of proton-neutron $\mathrm{T}=0$ correlations, 


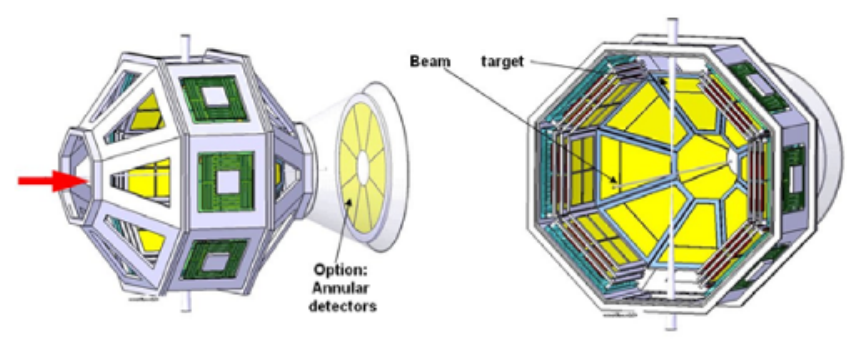

Figure 2. Barrel and trapezoidal design proposed for GASPARD composed of three-stage Silicon telescopes and Front End Electronics close to the detector.

deuteron transfer can be seen an efficient tool [27]. Some work have been performed in that direction along the $\mathrm{N}=\mathrm{Z}$ line [28] but these studies are still marginal due to difficult interpretation of the absolute cross sections in terms of proton-neutron correlations.

In all these approaches, the effect of final state interactions on the observables is central and should be further investigated. To address this question, a careful benchmarking program of all relevant technics on the same physics case and, if possible, at different incident energies seems unavoidable.

\section{New detection systems dedicated to direct reactions with radioactive beams}

The construction of new facilities or upgrade of existing machines dedicated to radioactive-ion beam studies parallels the development of a new instrumentation with increased sensitivity to probe the spectroscopy of the most exotic nuclei. This increase in sensitivity can be obtained via a higher luminosity, a larger energy range of detection and a better energy resolution, alternatively an efficient combination of several probes, such as gamma and particle spectroscopy. In most cases, each of these developments is adapted to the accelerators and hence to given incident energy regime, typically low energies for transfer reactions (GANIL, REX-ISOLDE, FRIB,...) or relativistic energies for quasifree scattering like experiments (RIKEN, FAIR,...).

At low incident energy, the European community has strongly pursued two directions in view of SPIRAL2: the ACTAR Time Projection Chamber [29] as a follow up of the MAYA detector [30] first developed at GANIL and the GASPARD Si-based telescope array [31] compact enough to fit inside a photon-detection array such as PARIS [32] or AGATA [33] partly issued from the MUST2-array developments [34]. These instrumentations were primarily developed to be used at SPIRAL2 and HIE-ISOLDE for transfer and inelastic scattering experiments. These new devices are complemented by the development of the very thin pure hydrogen target CHyMENE [35].

In the US, a complementary approach is being followed and focused on the use of magnetic field. The leading projects focusing on transfer and inelastic scattering studies are AT-TPC [36], a TPC located inside a solenoid, and HELIOS [37], a novel setup using Si detectors inside a solenoid that allows an unreached energy resolution when very thin targets are used. A cautious analysis of the reason(s) why Europe is not pursuing such a direction should be undertaken. 


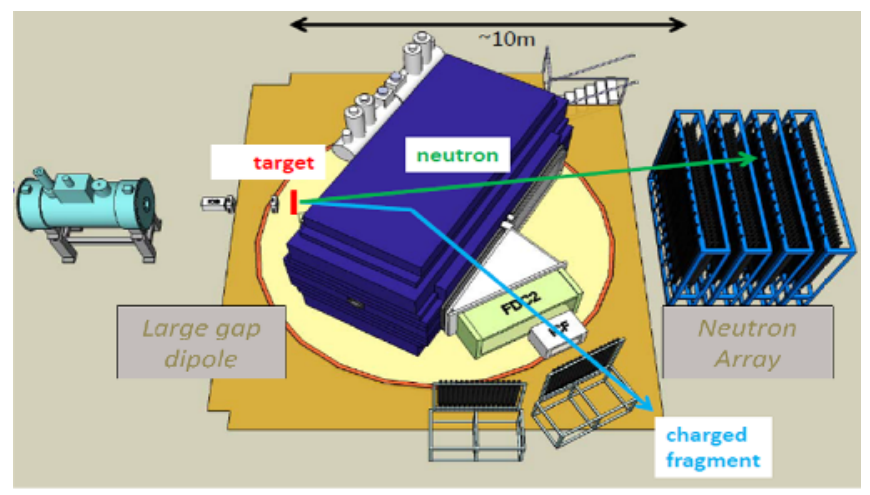

Figure 3. Schematic view of the SAMURAI setup in a configuration dedicated to the measurement of the full kinematic of nucleon knockout reactions. This setup can avantageously be combinded with the DALI2 $\gamma$-array for prompt gamma sepctroscopy and the thick MINOS hydrogen target for very rare events.

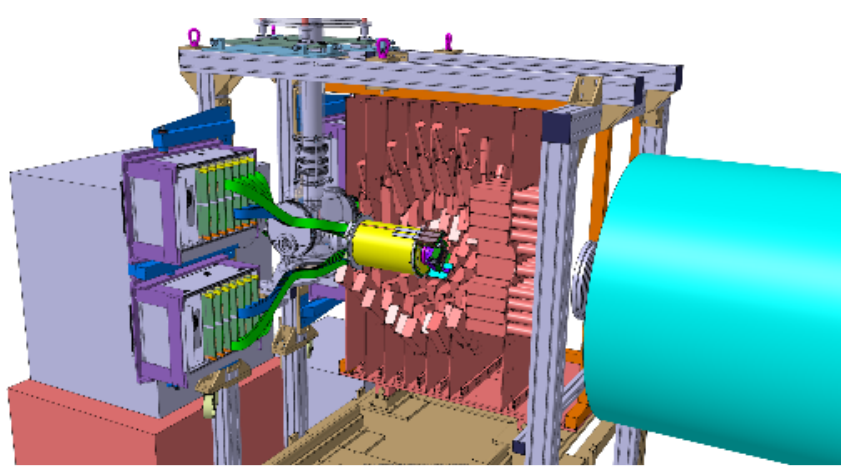

Figure 4. Schematic view of the MINOS target and TPC vertex tracker in position inside the DALI2 spectrometer for in-beam gamma spectrscopy at RIBF, RIKEN.

At relativistic energies, several large-acceptance spectrometers and associated detection have been or are being developed for direct reactions at new and future facilities: SAMURAI [38, 39] at RIBF, R3B [40] at FAIR. New generation scintillator arrays with high granularity, currently under study, should bring in a not so distant future a higher efficiency and enhanced resolution.

In-beam gamma spectroscopy should gain at least an order of magnitude, depending on the setup considered, with the use of the H2 target - vertex tracker MINOS [41].

Several of the above examples require digital electronics with triggers rates above a $\mathrm{kHz}$ or with strong software-based event rejection capabilities. The GET electronics (General Electronics for TPCs) has been recently developed for such purposes within a collaboration composed of CEA, IN2P3 (France) and NSCL (US).

It is believed that direct reactions with hydrogen will provide in the future, benchmark data for the understanding of nuclear dynamics at both low energy and relativistic energy. Indeed, in view of 
more reliable results from reaction analysis, one may advocate that hydrogen induced reactions, when applicable to a specific study, should be favored to heavy targets whose structure can only add more complexity in the system [42].

On the theory side, a more consistent treatment of nuclear structure and the reaction mechanism is required. Such consistent calculations at an ab initio level are today performed for light systems at very low incident energy. Such brute force approach is considered inadequate for transfer or knockout.

\section{Summary and outlook}

Direct reactions have provided the physics community at large a sizeable part of the information required to reach our present understanding of the nuclear shell structure. Shell evolution with isospin and in-medium correlations can be effectively investigated via transfer and fast nucleon removal. It is considered here that direct reactions will be a corner-store of studies performed at upcoming newgeneration radioactive beam facilities.

The study of highly exotic nuclei with very low binding energy requires a careful selection of approaches, a handful of which were initially developed for stable nuclei. Further, even though not seen as very appealing for structure studies, a proper benchmark for reaction models seems unavoidable to estimate the theoretical uncertainties. Redundancy of a measurement at two different incident energies and the systematical use of two different experimental techniques are possible directions to follow. In that sense, a LINAC-based machine with a rather easy change of incident energy should be a definite asset.

The community is developing several innovative detectors dedicated to direct reactions. They have distinct advantages, some improve the detection thresholds and allow the measurement of low-energy recoil reactions while others increase the compactness of the system and allow the combination of gamma and particle detection with high efficiency.

The near future should not be held within what is already under development. Colliders with exotic beams should offer new probes for nuclear structure. (e,e'p) reactions with exotic nuclei, as a follow up of the (e,e') SCRIT [43] programme at RIKEN, or nucleon annihilation from the interaction with antiprotons could be seen as a possible and exciting future possibilities where both new nuclear reaction models with innovative technical developments will allow a deeper understanding of nuclear mechanisms and structure.

Part of the present material has prepared for a review work made for the third EURISOL Users' Group Topical meeting [44]. The author would like to thank deeply F. Flavigny for providing Fig. 1 of this proceeding.

\section{References}

[1] Direct Nuclear Reactions, G. R. Satchler, Oxford University Press, 1983.

[2] C. Barbieri, D. Rohe, I. Sick, L. Lapikas, Phys. Lett. B 608, 47-52 (2005).

[3] G.J. Kramer, H.P. Blok, L. Lapikas, Nucl. Phys. A 679, 267 (2001).

[4] M. Baranger, Nucl. Phys. A 149, 225 (1970).

[5] R. J. Furnstahl, H.-W. Hammer, Phys. Lett. B 531, 203 (2002).

[6] T. Duguet, G. Hagen, Phys. Rev. C 85, 034330 (2012).

[7] P. Martin et al., Nucl. Phys. A 185, 465 (1972).

[8] Y. Uozumi et al., Nucl. Phys. A 576, 123 (1994). 
[9] Y. Uozumi et al., Phys. Rev. C 50, 263 (1994).

[10] A. Gade et al., Phys. Rev. C 77, 044306 (2008).

[11] J. Lee et al., Phys. Rev. Lett. 104, 112701 (2010).

[12] M. B. Tsang et al., Phys. Rev. Lett. 102, 062501 (2009).

[13] B.P. Kay, J.P. Schiffer, S.J. Freeman, accepted in Phys. Rev. Lett. (2013).

[14] F. Flavigny et al., Phys. Rev. Lett. 108, 252501 (2012).

[15] C. Louchart et al., Phys. Rev. C 83, 011601 (2011).

[16] F. Flavigny et al., Phys. Rev. Lett. 110, 122503 (2013).

[17] P. D. Duval, D. Goutte, M. Vergnes, Phys. Lett. B 124, 297 (1983).

[18] I. Tanihata et al., Phys. Rev. Lett. 100, 192502 (2008).

[19] G. Potel, F. Barranco, E. Vigezzi, R. A. Broglia, Phys. Rev. Lett. 105, 172502 (2010).

[20] G. Potel et al., Phys. Rev. Lett. 107, 092501 (2011).

[21] K. Yoneda et al., Physics Letters B 499, 233-237 (2001).

[22] K. Yoneda et al., Phys. Rev. C 74, 021303 (2006).

[23] E.C. Simpson, J.A. Tostevin, Phys. Rev. C 79, 024616 (2009).

[24] K. Wimmer et al., Phys. Rev. Lett. 109, 202505 (2012).

[25] D. Lacroix, J.-A. Scarpaci, Ph. Chomaz, Nucl. Phys. A 658, 273 (1999).

[26] M. Assié, D. Lacroix, Phys. Rev. Lett. 102, 202501 (2009).

[27] P. Van Isacker, D. D. Warner, A. Frank, Phys. Rev. Lett. 94, 162502 (2005).

[28] P. Fröbich, Phys. Lett. B 37, 338 (1971).

[29] http://pro.ganil-spiral2.eu/spiral2/instrumentation/actar-tpc

[30] C.E. Demonchy et al., Nucl. Instr. Meth. in Res. Phys. A 573, 145 (2007).

[31] http://gaspard.in2p3.fr/index.html

[32] http://paris.ifj.edu.pl

[33] S. Akkoyun et al., Nucl. Instr. Meth. in Res. Phys. A 688, 26 (2012).

[34] E.C. Pollacco et al., Nucl. Instr. Meth. in Res. Phys. A 421, 471 (1999).

[35] A. Gillibert et al., to be published in Eur. Phys. Jour. A (2013).

[36] http://www.nscl.msu.edu/exp/sr/attpc

[37] A. H. Wuosmaa et al., Nucl. Instr. Meth. in Res. Phys. A 580, 1290 (2007).

[38] Technical proposal, http://rarfaxp.riken.go.jp/RIBF-TAC05/10_SAMURAI.pdf

[39] Y. Shimizu et al., Journal of Physics, Conference Series, 312, 052022 (2011).

[40] Technical Proposal, http://www-win.gsi.de/r3b/Documents/R3B-TP-Dec05.pdf

[41] MINOS, European Research Council Starting Grant - 258301 (2010-2015); A. Obertelli et al., in preparation (2013).

[42] A. Obertelli, T. Uesaka, Eur. Phys. Jour. A 47, 105 (2011).

[43] T. Suda et al., Phys. Rev. Lett. 102, 102501 (2009).

[44] "Physics of light Exotic Nuclei", Lisbon, Portugal, 2012, Ed. by Angela Bonaccorso, Lidia Ferreira and Bjorn Jonson. 
